Yaroslavl State University

Preprint YARU-HE-97/01

hep-ph/9702284

\title{
Electromagnetic Catalysis of the Radiative Decay of the Axion
}

\author{
N.V. Mikheev \\ Division of Theoretical Physics, Department of Physics, \\ Yaroslavl State University, Yaroslavl 150000, Russia \\ E-mail: mikheev@yars.free.net \\ and \\ L.A. Vassilevskaya \\ Moscow Lomonosov University, V-952, Moscow 117234, Russia \\ E-mail: vasilevs@vitep5.itep.ru
}

\begin{abstract}
The radiative decay of the axion $a \rightarrow \gamma \gamma$ is investigated in an external electromagnetic field in DFSZ model in which axion couples to both quarks and leptons at tree level. The decay probability is strongly catalyzed by the external field, namely, the field removes the main suppression caused by the smallness of the axion mass.
\end{abstract}

will be published in Phys. Lett. B 
Various extensions of the Standard Model (SM) predict the existence of light neutral pseudoscalars which are the subject of constant theoretical and experimental studies. The axion [1, 2] is the most widely discussed pseudoscalar particle proposed by R.Peccei and H.Quinn to solve a major theoretical problem of CP-conservation in strong interactions. Although the original axion, associated with Peccei-Quinn $(P Q)$ symmetry breaking at the weak scale $\left(f_{w}\right)$, is excluded experimentally, many variant $P Q$ models and their associated axions are of great interest (see, for example [3, 田). If the breaking scale of $P Q$ symmetry $f_{a} \gg f_{w}$ (the latest astrophysical data yield $f_{a} \geq 10^{10} \mathrm{GeV}$ ), the resulting, so called, invisible axion is very light $\left(m_{a} \sim f_{a}^{-1}\right.$ ) and very weakly coupled (coupling $\sim f_{a}^{-1}$ ). At present astrophysical and cosmological considerations leave a rather narrow window for the axion mass [3, 5, 6]:

$$
10^{-6} \mathrm{eV} \leq m_{a} \leq 10^{-3} \mathrm{eV} .
$$

A survey of various processes involving axion production and astrophysical methods for obtaining constraints on the parameters of axion models is given in [3]. Unfortunately a problem of its possible existence which is very important for the theoretical description of elementary particles remains unresolved. It can be naturally explained by the fact that the axion lifetime in vacuum is very large [3]:

$$
\tau(a \rightarrow 2 \gamma) \sim 6,3 \cdot 10^{48} s\left(\frac{10^{-3} e V}{m_{a}}\right)^{6}\left(\frac{E_{a}}{1 M e V}\right) .
$$

On the other hand, one can get more precise constraints on weakly coupled particles properties with an influence of external electromagnetic fields taken into account. It is also important in some astrophysical and cosmological considerations to take strong external fields (of order the critical, so called, Schwinger value $F_{s}=m_{e}^{2} / e \simeq 0.44 \cdot 10^{14} G$ ) into account. Notice that the decay probability in an external field depends on not only typical kinematical invariants of type $m^{2}, p^{2}$, but field invariants $\left|e^{2}(F F)\right|^{1 / 2},\left|e^{2}(\tilde{F} F)\right|^{1 / 2},\left|e^{2}(p F F p)\right|^{1 / 3}$ as well (here $m$ and $p_{\mu}$ are the mass and the 4 -momentum of the particles, $F_{\mu \nu}$ is the external field tensor, $\left.p F F p=p^{\mu} F_{\mu \nu} F^{\nu \rho} p_{\rho}\right)$. In ultrarelativistic limit the field invariant $\left|e^{2}(p F F p)\right|^{1 / 3}$ can occur the largest one. This is due to the fact that in the relativistic particle rest frame the field may turn out to be of order of the critical one or even higher, appearing very close to the constant crossed field $(\vec{E} \perp \vec{H}, E=H)$, where $(F F)=(F \tilde{F})=0$. Thus, the calculation in this field is the relativistic limit of the calculation in an arbitrary relatively smooth field, possesses a great extent of generality and acquires interest by itself.

In this work we study the double radiative axion decay $a \rightarrow \gamma \gamma$ in the external crossed field.

We consider Dine-Fischler-Srednicki-Zhitnitskii (DFSZ) axions [7] which couple to both quarks and leptons at tree level. The corresponding interaction lagrangian has the form:

$$
\mathcal{L}_{a f}=-i g_{a f}\left(\bar{f} \gamma_{5} f\right) a
$$

where $g_{a f}=C_{f} m_{f} / f_{a}$ is a dimensionless Yukawa coupling constant, $C_{f}$ is a model-dependent factor, $m_{f}$ is a fermion mass. 


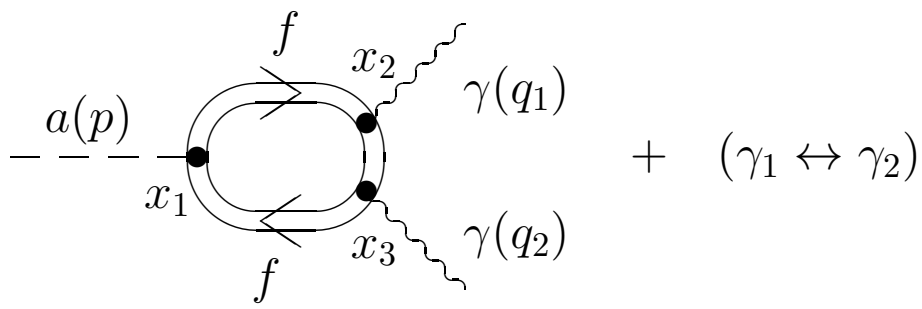

Figure 1.

A matrix element of $a \rightarrow \gamma \gamma$ decay is described by two three-point loop diagrams (Fig.1) where double lines imply that the influence of the external field in the propagators of virtual fermions is taken into account exactly. An expression for the matrix element in the external field is:

$$
\begin{aligned}
S & =\frac{e_{f}^{2} g_{a f}}{\sqrt{2 E_{a} V \cdot 2 \omega_{1} V \cdot 2 \omega_{2} V}} \int d^{4} x_{1} d^{4} x_{2} d^{4} x_{3} \\
& \times \exp \left\{-i p x_{1}+i q_{1} x_{2}+i q_{2} x_{3}\right\} \\
& \times S p\left\{S^{(F)}\left(x_{1}, x_{3}\right)\left(\varepsilon_{2} \gamma\right) S^{(F)}\left(x_{3}, x_{2}\right)\left(\varepsilon_{1} \gamma\right) S^{(F)}\left(x_{2}, x_{1}\right) \gamma_{5}\right\} \\
& +\left(\varepsilon_{1}, q_{1} \leftrightarrow \varepsilon_{2}, q_{2}\right)
\end{aligned}
$$

Here $e_{f}=e Q_{f}, e>0$ is the elementary charge, $Q_{f}$ is a relative fermion charge in the loop; $q_{1}, q_{2}$ and $\varepsilon_{1}, \varepsilon_{2}$ are the 4-momenta of the final photons and their polarization 4-vectors, respectively; $p$ is the 4-vector of the decaying axion; $(\varepsilon \gamma)=\left(\varepsilon^{\mu} \gamma_{\mu}\right), \gamma_{\mu}, \gamma_{5}$ are Dirac $\gamma$-matrices.

The expression for the propagator of a charged fermion in the crossed field $S^{(F)}(x, y)$ in the proper time formalism has the form:

$$
\begin{aligned}
S^{(F)}(x, y) & =e^{i \Omega(x, y)} S(X), \\
\Omega(x, y) & =-e_{f} \int_{y}^{x} d \xi_{\mu}\left[A_{\mu}(\xi)+\frac{1}{2} F_{\mu \nu}(\xi-y)_{\nu}\right], \\
S(X) & =-\frac{i}{16 \pi^{2}} \int_{0}^{\infty} \frac{d s}{s^{2}}\left[\frac{1}{2 s}(X \gamma)-\frac{i e_{f}}{2}(X \tilde{F} \gamma) \gamma_{5}\right. \\
& \left.-\frac{s e_{f}^{2}}{3}(X F F \gamma)+m_{f}+\frac{s m_{f} e_{f}}{2}(\gamma F \gamma)\right] \\
& \times \exp \left[-i\left(s m_{f}^{2}+\frac{X^{2}}{4 s}+\frac{s e_{f}^{2}}{12}(X F F X)\right)\right],
\end{aligned}
$$

where $X=x-y, m_{f}$ and $e_{f}$ are the mass and the charge of the intermediate fermion; $F_{\mu \nu}$ and $\tilde{F}_{\mu \nu}=(1 / 2) \varepsilon_{\mu \nu \alpha \beta} F_{\alpha \beta}$ are the tensor and the dual tensor of the constant crossed field; $A_{\mu}$ is the four-potential. 
Calculations of some processes amplitudes describing by three-point loop diagrams are very complicated 1 and the general expression for the $a \gamma \gamma$-vertex has a cumbersome form and will be published elsewhere.

The field-induced contribution $\Delta M=M-M_{0}$ to the amplitude $M\left(M_{0}\right.$ is the vacuum amplitude) of the ultrarelativistic axion decay $\left(E_{a} \gg m_{a}\right)$ we have obtained is:

$$
\begin{aligned}
\Delta M \cong \frac{\alpha}{\pi} \sum_{f} \frac{Q_{f}^{2} g_{a f}}{m_{f}^{5}}\left[\frac{e_{f}^{2}}{1-\lambda}\left(f_{1} \tilde{F}\right)\left(f_{2} F\right) J+O\left(\frac{m_{a}}{E_{a}}\right)+\left(\varepsilon_{1}, q_{1} \leftrightarrow \varepsilon_{2}, q_{2}\right)\right], \\
J=-\int_{0}^{1} d x \int_{0}^{1-x} d y y(1-y-2 x) \eta^{3} \frac{d^{2} f(\eta)}{d \eta^{2}}, \\
f(\eta)=i \int_{0}^{\infty} d t \exp \left[-i\left(\eta t+\frac{1}{3} t^{3}\right)\right], \\
\eta=\left(\chi^{2} Z(x, y, \lambda)\right)^{-1 / 3}, \\
Z(x, y, \lambda)=-2 x y\left(1-3 x-3 y+2 x^{2}+2 y^{2}+3 x y\right) \lambda(1-\lambda) \\
\chi^{2}=\frac{x_{f}^{2}(1-x)^{2}(1-\lambda)^{2}+y^{2}(1-y)^{2} \lambda^{2}}{m_{f}^{6}}, \\
f_{i \alpha \beta}=q_{i \alpha} \varepsilon_{i \beta}-q_{i \beta} \varepsilon_{i \alpha}, \quad i=1,2,
\end{aligned}
$$

where $\lambda=\omega_{1} / E_{a}$ is the first photon relative energy, $E_{a}$ is the decaying axion energy; $J$ is the integral of the Hardy-Stokes function $f(\eta), \chi$ is the, so called, dynamic parameter.

Using the standard methods we get an expression for the axion decay probability in the external field:

$$
W^{(F)}=\frac{1}{\pi E_{a}}\left(\frac{\alpha}{\pi}\right)^{2} \int_{0}^{1} d \lambda \lambda^{2}\left|\sum_{f} Q_{f}^{2} g_{a f} m_{f} \chi^{2} J\right|^{2},
$$

With the strong hierarchy of fermion masses the contribution of the fermion with the maximum value of the dynamic parameter $\chi^{2}$ can dominate in (7):

$$
W^{(F)} \cong 3.32\left(\frac{\alpha}{\pi}\right)^{2} \frac{\left(Q_{f}^{2} g_{a f} m_{f}\right)^{2}}{\pi E_{a}} P(\chi) .
$$

The numerical values of the function $P(\chi)$ are presented in Table and asymptotic behaviors for small and large values of the dynamical parameter $\chi$ are given:

\footnotetext{
${ }^{1}$ The history of calculations of an analogous three-point loop process of the photon splitting $\gamma \rightarrow \gamma \gamma$ in external electromagnetic fields goes back to the pioneer work by Adler [9] and it is still in progress [10].
} 


$$
\begin{aligned}
\left.P(\chi)\right|_{\chi \ll 1} & \simeq 2.31 \cdot 10^{-5} \cdot \chi^{8}\left(1+9.5 \chi^{2}+\cdots\right), \\
\left.P(\chi)\right|_{\chi^{1 / 3} \gg 1} & \simeq 1-\frac{8.8}{\chi^{1 / 3}}+\frac{32.0}{\chi^{2 / 3}}-\cdots
\end{aligned}
$$

Let us compare the probability (8) we have obtained with the well known axion decay probability in vacuum [3]:

$$
W^{(0)}=\frac{g_{a \gamma}^{2} m_{a}^{4}}{64 \pi E_{a}}
$$

Here $g_{a \gamma}=\left(\alpha / 2 \pi f_{a}\right)(E / N-1.92 \pm 0.08)$ [3], where $E$ and $N$ are the model-dependent coefficients of the electromagnetic and color anomalies. The comparison

$$
R_{f}=\frac{W^{(F)}}{W^{(0)}} \cong 2,12 \cdot 10^{2}\left(\frac{\alpha}{\pi}\right)^{2}\left(\frac{Q_{f}^{2} g_{a f} m_{f}}{g_{a \gamma} m_{a}^{2}}\right)^{2} P(\chi)
$$

illustrates the enhancing (catalyzing) influence of the external field on the ultrarelativistic axion decay $\left(E_{a} \gg m_{a}\right)$, because $W^{(F)}$ (8) does not contain a suppression associated with the smallness of the decaying axion mass. Notice that the analogous phenomenon of strong catalyzing effect of external electromagnetic field on rare loop process of neutrino radiative decay was discovered in [8]. For the electron $R_{e}$ has the form:

$$
R_{e} \simeq 10^{37}\left(\frac{\cos ^{2} \beta}{E / N-1.92}\right)^{2}\left(\frac{10^{-3} e V}{m_{a}}\right)^{4} P(\chi)
$$

Here $\cos ^{2} \beta$ determines the electron Yukawa coupling $g_{a e}=\frac{1}{3} \cos ^{2} \beta\left(m_{e} / f_{a}\right)$ in the DFSZ model. Large values of the dynamic parameter $\chi \gg 1$, when $P(\chi) \sim 1$, can be realized, for example, in case of the decaying axion energy $\sim 10 \mathrm{MeV}$ and the magnetic field strength $\sim F_{s}$. At present a possible existence of strong external fields $F \geq F_{s}$ is widely discussed in astrophysics, where strong magnetic fields can take place (a process of a coalescence of neutron stars, an explosion of a supernova of the type SN 1987A).

Note that the expressions for the amplitude (6) and the decay probability (8) can be applied to other pseudoscalar particles with the coupling of type (3), even though these particles are massless. This is due to the corresponding dispersion low in the magnetic field.

\section{Acknowledgements}

The authors thank V.A. Rubakov for fruitful discussions and M.E. Shaposhnikov and A.Ya. Parkhomenko for useful critical remarks. 


\section{References}

[1] R.D. Peccei and H.R. Quinn, Phys. Rev. Lett. 38 (1977) 1440; Phys. Rev. D16 (1977) 1791.

[2] S. Weinberg, Phys. Rev. Lett. 40 (1978) 223;

F. Wilczek, Phys. Rev. Lett. 40 (1978) 271.

[3] G.G. Raffelt, Phys. Rept. 198 (1990) 1.

[4] R.D. Peccei, Preprint hep-ph/9606475 (QCD, Strong CP and Axions).

[5] M.S. Turner, Phys. Rept. 197 (1990) 67.

[6] G.G. Raffelt, Stars as Laboratories for Fundamental Physics (University of Chicago Press: Chicago 1996).

[7] M. Dine, W. Fischler, and M. Srednicki, Phys. Lett. B 104 (1981) 199;

A.P. Zhitnisky, Sov. J. Nucl. Phys. 31 (1980) 260.

[8] A.A. Gvozdev, N.V. Mikheev, and L.A. Vassilevskaya, Phys. Lett. B289 (1992) 103; B321 (1994) 108; B323 (1994) 179; Phys. Rev. D54 (1996) 5674.

[9] S.L. Adler, Ann. Phys. N.Y. 67 (1971) 599.

[10] S.L. Adler and C. Schubert, Phys. Rev. Lett. 77 (1996) 1695;

V.N. Baier, A.I. Milstein, and R.Zh. Shaisultanov, Phys. Rev. Lett. 77 (1996) 1691. 
Table 1:

\begin{tabular}{|c|c|}
\hline$\chi$ & $P(\chi)$ \\
\hline $10^{-2}$ & $2.31 \cdot 10^{-21}$ \\
\hline $10^{-1}$ & $2.53 \cdot 10^{-13}$ \\
1 & $2.12 \cdot 10^{-5}$ \\
5 & $4.68 \cdot 10^{-3}$ \\
10 & $1.7 \cdot 10^{-2}$ \\
100 & 0.17 \\
$10^{3}$ & 0.44 \\
$10^{4}$ & 0.66 \\
$10^{6}$ & 0.871 \\
$\infty$ & 1 \\
\hline
\end{tabular}

